\title{
El Inventario de los Cinco Grandes Factores de Personalidad en el contexto argentino: puesta a prueba de los factores de orden superior*
}

\section{The Big Five Personality Inventory in the Argentinean Context: Testing Higher Order Factors}

\author{
Gabriel Genise** \\ ORCID: 0000-0002-6411-4141 \\ Universidad de Flores, Argentina \\ Joaquín Ungaretti \\ ORCID: 0000-0003-1185-9139 \\ Edgardo Etchezahar \\ ORCID: 0000-0002-3289-194X \\ Universidad de Buenos Aires, Conicet, \\ Argentina \\ Recibido: 13 de noviembre de 2019 \\ Revisado: 17 de febrero de 2020 \\ Aceptado: 22 de abril de 2020
}

\section{Resumen}

El Modelo de los Cinco Factores (FFM) de la personalidad predomina en la literatura científica y el Inventario de los Cinco Grandes (BFI) constituye una de sus principales evaluaciones. Considerando las limitaciones de estudios previos, el objetivo del presente artículo fue explorar la dimensionalidad del instrumento de evaluación BFl, evaluar los factores de orden superior y analizar sus relaciones con la autoestima, la edad y el sexo de los participantes. Participaron 549 estudiantes universitarios. Los resultados indicaron adecuadas propiedades psicométricas para el BFI y para el modelo de dos factores de orden superior. Además, se observaron diferencias significativas según el sexo en amabilidad y neuroticismo, relaciones entre edad, neuroticismo y responsabilidad, así como también relaciones entre ambos factores de orden superior y la autoestima.

Palabras clave: BFI, FFM, orden superior, personalidad, validación.

Artículo de investigación. Citar como: Genise, G., Ungaretti, J. y Etchezahar, E. (2020). El Inventario de los Cinco Grandes Factores de Personalidad en el contexto argentino: puesta a prueba de los factores de orden superior Diversitas: Perspectivas en Psicología, 16(2), 325-340. https: //doi.org/10.15332/22563067.6298

Autor de correspondencia: Dr. Gabriel Genise, PsyD en Psicología, Especialista en Terapia Cognitiva. Correo electrónico: genisegp@gmail.com. 


\section{Abstract}

The Five-Factor Model (FFM) of personality predominates in the scientific literature, and the Big Five Inventory (BFI) is one of its main evaluations. Considering the limitations of previous studies, the aim of the present study was to explore the dimensionality of the $\mathrm{BFI}$ assessment instrument, to test higherorder factors and to analyze its relations with self-esteem, age, and sex of the participants. A total of 549 university students participated. The results showed adequate psychometric properties for the BFI and for the two-factor model of higher order personality traits. In addition, significant differences were observed by sex in terms of agreeableness and neuroticism, relationships between age, neuroticism, and conscientiousness, as well as relationships between both higher order factors and self-esteem.

Keywords: BFI, FFM, higher order, personality, validation.

\section{Introducción}

El estudio de la personalidad desde la psicológica se ha trabajado a partir de diversos marcos teóricos, entre los cuales se destacan la teoría genética de la personalidad (Penke, Denissen y Miller, 2007), la teoría psicodinámica (Carver y Scheier, 2004), la teoría comportamental (Phelps, 2000), la sociocognitiva (Mischel, 1979), la humanista (Snygg, 1949), la biopsicosocial (Macmillan, 2000) o la teoría de los rasgos (Santrock, 2008). Esta multiplicidad de perspectivas teóricas para el abordaje de la personalidad ha dificultado el establecimiento de una conceptualización única de dicho constructo. Sin embargo, la teoría de los rasgos ha sido, entre los diferentes enfoques, el más estudiado en el área de la Psicología de la Personalidad (McCrae y Costa Jr, 2012), siendo el Modelo de los Cinco Factores (FFM, por sus siglas en inglés; Costa y McCrae, 1980) uno de los que ha generado mayor consenso en la literatura científica.

\section{El Modelo de los Cinco Factores de la Personalidad (FFM)}

Si bien el FFM comienza a ser teorizado como tal en 1980, sus raíces se remontan a 1930, década en la que varios investigadores utilizaron el lenguaje natural como una fuente de atributos en la taxonomía científica (Ashton, Lee, Goldberg y de Vries, 2009). El comienzo de estos trabajos se dio a partir de la extracción de todos los términos relevantes de la personalidad del diccionario utilizando un enfoque léxico. Este enfoque postula que la mayoría de las características de la personalidad con relevancia social fueron codificadas en el lenguaje natural (John, Robins y Pervin, 2008). En esta línea, Allport y Odbert (1936) llevaron a cabo un estudio léxico sobre la personalidad incluyendo todos los términos del diccionario que podrían haber sido utilizados como distintivos del comportamiento de un ser humano hacia otro. Años más tarde, Catell (1943) retomó los aportes de Allport y Odbert (1936) utilizando como procedimiento de selección de términos el enfoque semántico y empírico, logrando reducir los rasgos iniciales a 12 dimensiones que luego se incluyeron en su cuestionario de 16 factores de personalidad (Genise, 2015). Más adelante, Costa y McCrae (1980) observaron en estos trabajos que la multiplicidad de rasgos que caracterizaban a los individuos y que les permitían diferenciarse de los demás, podrían ser agrupados en cinco grandes factores: Apertura a la Experiencia ( 0 ), Responsabilidad (C), Extraversión (E), Amabilidad (A) y Neuroticismo (N).

De acuerdo con los autores, la apertura a la experiencia se caracteriza por la presencia de imaginación activa, sensibilidad estética, capacidad de introspección o curiosidad intelectual. Por su parte, la responsabilidad se refiere a la capacidad de ciertos individuos para controlar los impulsos, actuar con propósitos o metas claras, planificar, organizar 
y llevar adelante proyectos e ideas (Lingjaerde, Regine Foreland y Engvik, 2001), mientras que la Extraversión evalúa la tendencia a comunicarse con las demás personas, a ser asertivos, activos y verbalizadores. Por último, la amabilidad caracteriza a quienes tienen la capacidad para establecer vínculos psicosociales, muestran comportamientos altruistas y una amplia disposición a preocuparse por los demás, al tiempo que el Neuroticismo implica inestabilidad emocional o predisposición a experimentar emociones de valencia negativa como miedos, sentimientos de culpa, tristeza o enojo (Lingjaerde et al., 2001).

El FFM cuenta con distintos instrumentos para su evaluación entre los que se destacan el Inventario de Personalidad NEO (NEO-PI-R; Costa y MC Rae, 1992), el IPIP NEO (Goldberg, 1999), el Inventario de los Cinco Factores neo (NEO FFI; Costa y MC Rae, 1992), el Inventario de los Cinco Grandes Factores de Personalidad (BFl; John, Donahue y Kentle, 1991), el Inventario de Personalidad de Diez Ítems (T1PI; Goldberg y Rosolack, 1994) o el Inventario de Personalidad de Cinco Ítems (FIPI; Ashton et al., 2009). Muchas de estos instrumentos han sido evaluados y adaptados en más de cincuenta países, con traducciones a más de diez idiomas, observando en todos los casos adecuadas propiedades psicométricas que permiten sostener empíricamente la teoría que subyace al FFM (Benet Martinez y John, 1998; Chan et al., 2012; John, Naumann y Soto, 2008; Schmitt, Allik, McCrae y Benet-Martínez, 2007).

De los instrumentos de evaluación mencionados, el BFI se ha destacado a partir de dos grandes aspectos considerados por John et al. (1991) para su construcción original. Por un lado, se presenta como una técnica breve que permite una evaluación eficiente de las cinco dimensiones de personalidad cuando no es necesaria una diferenciación más exhaustiva de las facetas individuales. Por otra parte, otro de los aspectos positivos del BFI que proporciona mayor consistencia en las respuestas radica en el uso de frases cortas y no simplemente en adjetivos (Goldberg y Kilkowski, 1985). A diferencia del resto de los instrumentos para la evaluación del FFM, se ha señalado que el BFı brinda mayor contextualización que las respuestas basadas solamente en adjetivos, a la vez que menor complejidad que el formato de ítems presentes en las diferentes variantes del instrumento NEO (John y Srivastava, 1999). Además, el BFI ha sido estudiado de manera sistemática con diferentes variables psicosociales como el prejuicio (Ekehammar, Akrami, Gylje y Zakrisson, 2004), la religiosidad (Saroglou y Muñoz García, 2008) y los valores sociales (Caprara, Schwartz, Capanna, Vecchione y Barbaranelli, 2006).

En el contexto argentino se han realizado diversas adaptaciones y validaciones de instrumentos que permiten evaluar el FFM, tales como el inventario de personalidad IPIP NEO (Cupani, Pilatti, Urrizaga, Chincolla y Richaud de Minzi, 2014), así como también el IPIP-FFM (Cupani, 2009). En cuanto al BFI, uno de los primeros trabajos en utilizarlo fue el desarrollado por Castro Solano y Casullo (2001). Los autores realizaron una retrotraducción de los ítems de la versión del instrumento de John (1990), e informaron la consistencia interna de cada uno de los factores. Analizaron sus relaciones con el bienestar psicológico y el rendimiento académico en adolescentes argentinos de 13 a 19 años. La consistencia interna de cada dimensión fue adecuada (Extraversión: $a=0.67$; Amabilidad: $a=0.67$; Responsabilidad: $a=0.74$; Neuroticismo: $a=0.68$; Apertura a la experiencia: $a=0.72$ ). En cuanto a la estructura de cinco factores explicaba el $40 \%$ de la varianza de las puntuaciones. Años más tarde, Castro Solano (2005) analizó las propiedades psicométricas del BFI en población universitaria civil y militar. Nuevamente la consistencia interna de cada uno de los factores fue adecuada, tanto para la población de cadetes militares (Extraversión: $a=0.71$; Agradabilidad: $a=0.55$; Responsabilidad: $a=0.69$; Neuroticismo: $a=0.73$; Apertura a la experiencia: $a=0.69$ ), como para la población civil (Extraversión: $a=0.68$; Agradabilidad: $a=0.66$; Responsabilidad: $a=0.70$; Neuroticismo: $a=0.74$; Apertura a la experiencia: $a=0.77)$. Además, se mostró su validez de constructo tras realizar un análisis factorial exploratorio observando una estructura compuesta por cinco factores que explicaron el $45 \%$ de la varianza de las puntuaciones.

A partir del aporte realizado en los estudios mencionados en el estudio de la personalidad en el contexto argentino, se pudieron identificar una serie de inconvenientes con diferentes ítems de la validación 
argentina del instrumento (Castro Solano y Casullo, 2001). Por ejemplo, se observó que ciertos ítems que presentaron cargas factoriales adecuadas en la dimensión a la que pertenecen, también obtuvieron elevadas cargas en otros factores (e.g. ítem 16). Asimismo, se observaron ítems con alta saturación en un factor que no era el adecuado y baja saturación en el propio (e.g. ítem 27). Por último, también se observó que ciertos ítems (e.g., 26) presentaron elevadas cargas en otros factores y valores no significativos en el propio.

\section{Factores de orden superior en el FFM}

Con el objetivo de organizar la evidencia empírica en torno al FFM, Digman (1997) realizó un metaanálisis sobre la base de 14 estudios en los que se había utilizado dicho modelo. El autor observó que los cinco grandes factores de la personalidad se encontraban agrupados por otros dos factores de orden superior, a los que denominó Alpha (compuesto por los factores Amabilidad, Neuroticismo y Responsabilidad) y Beta (compuesto por los factores Extraversión y Apertura). De este modo, propuso que el factor Alpha podría comprenderse como una amplia colección de rasgos socialmente deseables: mientras que la hostilidad, el neuroticismo y la negligencia son rasgos indeseables en cualquier sociedad, la amabilidad, la estabilidad emocional y la responsabilidad son características que casi todas las personas quieren poseer (Digman, 1997). De acuerdo con Digman (1997), el factor Alpha podría ser consecuencia del proceso de socialización, ya que es durante dicho proceso que los niños aprenden a redirigir sus impulsos hostiles para descargar la agresión de un modo socialmente aceptable y así cumplir las exigencias sociales de "amabilidad". Del mismo modo, fallas o problemas en la socialización pueden favorecer comportamientos neuróticos, irresponsables o agresivos (Costa y Mc Rae, 1992). Por otra parte, el autor sostiene que el factor Beta se relacionaría con el crecimiento personal o la realización del sí mismo, es decir, estar abierto a toda experiencia y fomentar el desarrollo de la inteligencia. La extraversión y la apertura a la experiencia son los factores que presentan dichas características a través del ser arriesgado, buscar aventuras, ser proactivo, creativo, imaginativo, abierto a nuevas ideas y al cambio.
Posteriormente, DeYoung, Peterson y Higgins (2002) replicaron el modelo propuesto por Digman (1997) empleando dos muestras, una de 245 estudiantes universitarios y una de 222 sujetos en población general. Los autores observaron resultados similares a los reportados por Digman (1997), en donde se extrajeron dos factores de orden superior con valores superiores a uno, representando entre el $61 \%$ y el $63 \%$ de la varianza. Posteriormente, diseñaron un modelo de ecuaciones estructurales que reprodujo la estructura de dos factores de orden superior, a los que nombraron Estabilidad y Plasticidad. De Young et al. (2002) explicaron los factores de orden superior a partir del vínculo estrecho entre los rasgos de la personalidad y los aspectos biológicos: $\mathrm{El}$ factor "estabilidad" (Amabilidad, Responsabilidad, Neuroticismo) representa el medio por el cual los seres humanos se mantienen estables a través del sistema serotoninérgico rostral ascendente, mientras que el factor "plasticidad" (Apertura y Extraversión) refleja la tendencia a interactuar con estímulos nuevos (es decir, la plasticidad de la conducta y el pensamiento) a través del sistema dopaminérgico. Los autores también destacaron que estos factores no deben ser vistos como opuestos, sino como complementarios: Las personas deben poseer plasticidad para alcanzar estabilidad ante situaciones nuevas, al tiempo que la estabilidad brinda seguridad para enfrentar estas nuevas situaciones (DeYoung et al., 2002).

La estructura de dos factores de orden superior ha sido replicada por diferentes investigadores (e.g., Ashton et al., 2009; DeYoung, 2006; Musek, 2007; Rushton e Irwing, 2008, 2009) y en diferentes contextos como Alemania (Hirsh, 2010) Inglaterra (Woods y Hardy, 2011), Corea de Sur (Rushton, Bons y Hur, 2009) Jamaica (Hull y Beaujean, 2011), Suecia (Bäckström, Björklund y Larsson, 2009), o Turquía (Șimșek, 2012), identificándose relaciones específicas con diferentes variables psicológicas como la orientación vocacional (Rentfrow, Gosling y Potter, 2008), el comportamiento antisocial (Blackburn, Renwick, Donnelly y Logan, 2004), la conformidad (DeYoung et al., 2002), los valores sociales (Vecchione, Alessandri, Barbaranelli y Caprara, 2011), el bienestar (Șimșek y Koydemir, 2012) o la autoestima (Robins, Hendin y Trzesniewski, 2001; Shackelford y Michalski, 2011). 


\section{La autoestima y el Modelo de los Cinco Factores de la Personalidad (FFM)}

A pesar de que la autoestima y los rasgos de personalidad han sido explorados exhaustivamente de manera independiente, su investigación conjunta es relativamente reciente (Robins et al., 2001). De acuerdo con Robson (1989) la autoestima se define como el grado de aceptación que resulta de la evaluación que las personas hacen de su valía, atractivo, competencia y habilidad para satisfacer sus propias aspiraciones. Por su parte Rosenberg (1965), observa que una alta autoestima se encuentra relacionada al amor propio, al considerarse a sí mismo capaz de apreciar los méritos propios sin dejar de reconocer los errores. En contraposición, las personas con baja autoestima resultan más propensas a sentirse torpes, tímidas e incapaces de expresarse con confianza, por lo que se encuentran constantemente preocupadas por cometer un error o exponerse al ridículo (Lee y Hankin, 2009).

El análisis de la relación entre los rasgos de personalidad y la autoestima cobra particular relevancia puesto que la primera influye en la forma de percibirse y evaluarse a sí mismo, mientras que la segunda juega un papel fundamental en los procesos de conformación de la personalidad (McCrae y Costa, 2012). Prueba de esta relación han sido las investigaciones realizadas en diferentes contextos (Shackelford y Michalski, 2011), en las cuales generalmente se ha observado que la autoestima se encuentra fuertemente asociada al neuroticismo (Judge, Erez, Bono y Thoresen, 2002), moderadamente asociada a la extraversión y la responsabilidad (Digman, 1997; Paulhus y Reid, 1991) y débilmente asociada a la amabilidad y la apertura a la experiencia (Robins et al., 2001).

\section{Factores de orden superior de la personalidad y autoestima}

Recientemente, diferentes autores han observado que los factores de segundo orden de la personalidad se encontrarían asociados a la autoestima (Erdle, 2013; Simsek, 2012). Por ejemplo, Erdle y colaboradores (2009) exploraron esta relación en una muestra compuesta por 628.640 residentes estadounidenses que respondieron un cuestionario autoadministrable desde un sitio web, compuesto por el Inventario de los Cinco Grandes Factores de la Personalidad (BFl; John y Srivastava, 1999) y el Inventario de un ítem de Autoestima (SISE: Robins et al., 2001). Los resultados demostraron que tanto la estabilidad ( $r \geq 0.41)$, como la plasticidad $(r \geq 0.39)$, estaban asociadas a la autoestima, de modo que las personas con alta autoestima tendían a presentar una elevada estabilidad (amabilidad, estabilidad emocional, responsabilidad) y plasticidad (extroversión y apertura). De acuerdo con Erdle (2013), aquellos individuos con mayores niveles de estabilidad y plasticidad se sentían más a gusto consigo mismos y recibían mejor retroalimentación (feedback) del entorno, lo que probablemente conducía a una mayor autoestima. Resultados similares fueron reportados posteriormente por Simsek (2012), quien realizó un estudio en una población compuesta por 878 estudiantes universitarios turcos, administrando el $\mathrm{BFI}$ (John y Srivastava, 1999) y el Inventario de Diez Ítems de Autoestima de Rosenberg (RSEl; Rosenberg, 1965) observando que la autoestima presentó asociaciones con la estabilidad $(r \geq 0.51)$ y la plasticidad $(r \geq 0.41)$.

\section{Diferencias según la edad en los factores de personalidad}

Se han desarrollado dos teorías contrapuestas para explicar las diferencias en los rasgos de personalidad según la edad. Por una parte, aquella que explica estas diferencias a partir de aspectos genéticos universales de la especie y procesos intrínsecos de maduración (e.g., McCrae y Costa, 2012). De acuerdo con algunos representantes de esta teoría, los rasgos de personalidad alcanzan su madurez hacia los 30 años, con solo pequeños cambios luego de esa edad (Terracciano, Costa y McCrae, 2006). Por otra parte, las diferencias en el desarrollo de los rasgos de personalidad según la edad, ha sido explicada a partir de la compleja relación entre los procesos biológicos y las reacciones individuales a los cambios en el ambiente (Caspi y Roberts, 2001). Esta perspectiva sugiere que los cambios en la personalidad pueden ocurrir inclusive luego de los 30 años y no realiza predicciones explícitas sobre las 
diferencias en la magnitud de los cambios antes y después de los 30 años (Lucas y Donnellan, 2009).

Las investigaciones en esta línea han demostrado diferencias sistemáticas según la edad en los rasgos de personalidad, tanto en la adolescencia como en la adultez (e.g., Lucas y Donnellan, 2009; Srivastava, John, Gosling y Potter, 2003). Esto sugiere que durante la adolescencia los individuos tienden a aumentar los niveles de extraversión y apertura a la experiencia, y a disminuir en neuroticismo. Un estudio realizado por Soto y colaboradores (2008), tuvo como objetivo evaluar las hipótesis relacionadas con las diferencias en los rasgos de personalidad según la edad (entre los 10 y los 65 años) en grandes muestras de individuos a través de internet. Los autores hallaron una tendencia positiva en las relaciones entre la edad, los niveles de amabilidad y la responsabilidad. Por su parte, el neuroticismo demostró una tendencia negativa en la asociación entre los 25 años y la mediana edad, mientras que la extraversión mostró una pequeña tendencia negativa alrededor de los 25 años que luego se estabilizó en la edad adulta hasta la mediana edad. El análisis de la apertura a la experiencia demostró una tendencia positiva alrededor de los 25 años, desacelerándose hacia la mediana edad.

\section{Diferencias según el sexo en los factores de personalidad}

Las diferencias según el sexo en los rasgos de personalidad de los individuos han intentado ser explicadas desde modelos biológicos, socioculturales y biosociales (Feingold, 1994). Por ejemplo, las teorías de corte biologicistas, hacen hincapié tanto en las diferencias hormonales y sus efectos en el estado anímico y la personalidad, como en diferencias relacionadas con el sexo en las predisposiciones genéticas hacia condiciones psicopatológicas (Terracciano et al., 2006). La psicología evolutiva presupone que las diferencias según el sexo se originan a través de un proceso causal de selección sexual (Buss, 1995). Desde esta perspectiva, hombres y mujeres difieren en dominios en los que cada uno ha enfrentado diferentes problemas adaptativos a lo largo de la historia de la evolución humana. En contraste, los modelos socioculturales, postulan que los factores sociales y culturales producen directamente diferencias según el sexo en los rasgos de personalidad (e.g. Eagly, 1987). Finalmente, integrando las aproximaciones biológicas y sociales, Schmitt y colaboradores (2008) indicaron que las predisposiciones genéticas de personalidad de hombres y mujeres resultan sensibles a ciertos factores contextuales como por ejemplo el estrés ambiental, de modo tal que cada uno a su modo activa o suprime estas predisposiciones.

En esta línea, las investigaciones sobre las diferencias según el sexo en los rasgos de la personalidad, tanto en adolescentes como en adultos, reportaron resultados relativamente inconsistentes (e.g., Chapman, Lyness, Duberstein y Sörensen, 2007; Schmitt et al., 2008; Van Aken, Denissen, Branje, Dubas y Goossens, 2006). No obstante, en general se observó que son las mujeres quienes presentan una mayor tendencia a puntuar más alto en Neuroticismo y Amabilidad, no encontrándose diferencias significativas entre hombres y mujeres en el resto de los factores. Los autores sostienen que las diferencias observadas, se mantienen a lo largo de las diferentes etapas de la vida de los individuos (Chapman et al., 2007).

Teniendo en cuenta lo expuesto, si bien se han llevado a cabo estudios en el contexto argentino en donde se analicen las propiedades psicométricas del BFI (Castro Solano, 2005; Castro Solano y Casullo, 2001), se han detectado inconsistencias en sus ítems $y$, además, no se ha evaluado la existencia de factores de orden superior de la personalidad. Asimismo, tampoco se han realizado trabajos que indaguen en un mismo estudio las relaciones entre los cinco factores de la personalidad, el sexo, la edad y los niveles de autoestima de los participantes.

Partiendo de estas consideraciones, el propósito del presente estudio fue explorar la dimensionalidad del modelo FFM a través del BFI, para luego evaluar si el modelo de dos factores de orden superior era el que mejor se ajustaba a los datos. En segundo lugar, conocer las relaciones entre el modelo de los cinco grandes de la personalidad y la autoestima, para, finalmente, analizar si existen diferencias según el sexo en los rasgos de personalidad y en relaciones entre estos y la edad de los participantes. 


\section{Método}

\section{Participantes}

La selección de la muestra fue de tipo intencional, por cuotas de género y edad. Participaron 549 estudiantes universitarios residentes en la Ciudad de Buenos Aires, de los cuales el $25.9 \%$ fueron hombres $(n=142)$ y el $74.1 \%(n=407)$ mujeres. El rango etario de los participantes fue de 18 a 58 años $(M=25.25 ; D T=6.9)$. En cuanto a la ideología política de los participantes, se indagó en primer lugar si podían ubicarse en el continuo ideológico-político izquierda-derecha. Del total de los participantes, un $64.1 \%(n=352)$ respondió que sí, mientras que el $35.9 \%(n=197)$ respondió que no. De aquellos que pudieron ubicarse a sí mismos en dicho continuo, el 6.3\% $(n=23)$ se auto-posicionó en la extrema derecha, un $15.8 \%(n=58)$ en la centro-derecha, $38.4 \%(n=141)$ en el centro, $33.5 \%(n=123)$ en la centro-izquierda y un $6 \%(n=22)$ en la extrema izquierda. Además, el $32.4 \%(n=178)$ se consideraron a sí mismos muy religiosos, el $56.2 \%(n=309)$ algo religiosos y el $11.5 \%(n=62)$ nada religiosos.

\section{Procedimiento}

En la adaptación de la versión de la escala BFı al contexto argentino se siguieron los estándares metodológicos recomendados por la International Test Commission (ITC) para la adaptación de un instrumento de un contexto idiomático a otro (Muñiz y Hambleton, 2000). Si bien se contaba con traducciones previas de la técnica al idioma español (Castro Solano, 2005; Castro Solano y Casullo, 2001), las mismas no se adecuaban a los reactivos propuestos por los autores originales (John et al., 1991). Además, se procedió a la incorporación de ítems extra por cada dimensión, teniendo en cuenta las sugerencias realizadas por John et al. (1991), considerando una serie de ítems que, si bien quedaron por fuera de los 44 finales constituyeron el instrumento $\mathrm{BFI}$, obtuvieron cargas factoriales adecuadas $(>0.35)$ en el factor que los agrupaba, así como también muy bajas relaciones con el resto de los factores.

La administración del instrumento se llevó a cabo colectivamente al final de clases y resaltando que la participación de los sujetos en el estudio era de tipo voluntaria. Asimismo, se les comunicó que los datos obtenidos serían utilizados exclusivamente con fines científicos bajo la Ley Nacional 25.326 de protección de datos personales, resguardando el anonimato de los participantes.

\section{Instrumentos}

Los datos fueron recolectados a través de un instrumento de evaluación autoadministrable, asegurando el anonimato de los participantes. El mismo estuvo compuesto por las siguientes variables:

\section{Inventario de los Cinco Grandes Factores de la Personalidad (BFI)}

Se evaluaron los 44 ítems de la adaptación de la escala original (John et al., 1991) al contexto argentino más una batería de 10 ítems extra, dos por cada factor de la personalidad. Esto fue necesario atendiendo a los problemas observados en la carga factorial de los ítems presentados en versiones previas en español del BFI (Castro Solano y Casullo, 2001). La consistencia interna para cada uno de los factores del instrumento de evaluación original fueron adecuadas (Extraversión: $a=0.67$; Amabilidad: $a=0.67$; Responsabilidad: $a=0.74$; Neuroticismo: $a=0.68$; Apertura a la experiencia: $a=0.72$ ). Al igual que en la versión original y sus adaptaciones a diferentes contextos, se utilizó un formato de respuesta tipo Likert, con cinco anclajes en función del grado de acuerdo de los participantes, siendo 1 = "Completamente en desacuerdo" y 5 = "Completamente de acuerdo".

\section{Autoestima (RSES)}

Se utilizó la validación argentina (Góngora y Casullo, 2009) de la escala original de Rosenberg (1965), la cual consta de 10 ítems con cuatro opciones de respuesta que van desde 1 = "Extremadamente en desacuerdo", a 4 = "Extremadamente de acuerdo". La escala consta de cinco ítems directos y cinco invertidos. Las propiedades psicométricas del instrumento de evaluación resultaron adecuadas, tanto en su consistencia interna ( $a=0.81)$ como en su validez de constructo $(\mathrm{CFI}=0.94 ; \mathrm{AGFI}=93 ; \mathrm{RMSEA}=0.043$ ). 


\section{Variables socio-demográficas}

Se desarrolló un cuestionario ad-hoc para recabar este tipo de información, entre las variables consideradas se encontraban: sexo y edad.

\section{Análisis de datos}

El análisis de los datos se realizó mediante el paquete estadístico SPSS 20 y el programa EQS 6.1. Primero se calcularon los estadísticos descriptivos para cada ítem y luego se evaluó la consistencia interna de cada dimensión y la estructura factorial del instrumento. Posteriormente se analizó el grado de asociación de la escala BFI con otras variables en función de los antecedentes, para finalmente evaluar los factores de segundo orden de la personalidad a través de modelos de ecuaciones estructurales.

\section{Resultados}

\section{Análisis del Big Five Inventory (BFI)}

Inicialmente se llevó a cabo un análisis factorial exploratorio (AFE) de componentes principales con rotación Varimax ( $\mathrm{kMO}=0.807$; Esfericidad de Bartlett: $p>0.001$ ) basado en autovalores mayores a uno (según el scree test). Al igual que lo sugerido por la literatura, se obtuvieron cinco factores que representan lo planteado desde el FFM. En la matriz de componentes rotados se pudo observar que el ítem correspondiente a la dimensión Extraversión (26. Tiene una personalidad asertiva) y el ítem de la dimensión Amabilidad (27. Puede ser fría y distante) saturaron en factores que no corresponden según la teoría. Además, se hallaron bajas cargas factoriales en su dimensión de pertenencia en un ítem correspondiente a la dimensión Extraversión (16. Genera mucho entusiasmo) y en otro de la dimensión Amabilidad (22. Suele confiar en los demás). Asimismo, ambos ítems presentaron cargas factoriales cruzadas con otros factores. Se procedió a reemplazar estos cuatro ítems por otros, siguiendo las sugerencias de John et al. (1991). Se volvió a calcular un AFE ( $\mathrm{KMO}=0.808$; Esfericidad de Bartlett: $p<0.001$ ) con los 40 ítems originales sumados a los cuatro ítems extra. A continuación, se presenta la matriz de componentes rotados con la escala $\mathrm{BFI}$ definitiva (Tabla 1).

Tabla 1.

Matriz de componentes rotados de la escala BFI y alfa de Cronbach por cada factor

\begin{tabular}{lccccccc}
\hline & \multicolumn{7}{c}{ Factores } \\
\hline & $\mathbf{1}$ & $\mathbf{2}$ & $\mathbf{3}$ & $\mathbf{4}$ & $\mathbf{5}$ & ri-t & a-i \\
\hline Apertura a la experiencia $(\mathbf{a}=\mathbf{0 . 7 8})$ & & & & & & & \\
BF.25. Es inventiva & $\mathbf{0 . 7 3 8}$ & -0.137 & 0.163 & 0.052 & -0.030 & 0.572 & 0.747 \\
BF.20. Tiene mucha imaginación & $\mathbf{0 . 7 1 5}$ & 0.058 & 0.012 & 0.011 & 0.105 & 0.533 & 0.751 \\
BF.5. Es original. se le ocurren nuevas ideas & $\mathbf{0 . 6 5 5}$ & -0.031 & 0.193 & 0.081 & -0.071 & 0.499 & 0.756 \\
BF.15. Es ingeniosa. Profunda & $\mathbf{0 . 6 4 6}$ & 0.002 & 0.279 & 0.103 & -0.030 & 0.496 & 0.758 \\
BF.40. Le gusta reflexionar. jugar con las ideas & $\mathbf{0 . 6 2 1}$ & 0.036 & 0.096 & 0.000 & 0.057 & 0.505 & 0.755 \\
BFl.10. Siente curiosidad por muchas cosas diferentes & $\mathbf{0 . 5 5 4}$ & 0.012 & 0.100 & 0.066 & 0.133 & 0.418 & 0.766 \\
BFI.44. Tiene gustos sofisticados en arte. música o lite- - & $\mathbf{0 . 5 2 3}$ & -0.021 & -0.179 & -0.004 & -0.010 & 0.409 & 0.770 \\
ratura & & & & & & & \\
BFI.41. Tiene pocos intereses artísticos & $\mathbf{0 . 5 2 0}$ & -0.075 & -0.149 & 0.027 & 0.026 & 0.455 & 0.761 \\
BFI.30. Valora lo artístico. lo estético & $\mathbf{0 . 5 1 1}$ & 0.062 & -0.134 & -0.096 & 0.133 & 0.424 & 0.765 \\
BFI.35. Prefiere trabajos que son rutinarios & $\mathbf{0 . 2 9 5}$ & 0.034 & -0.047 & 0.203 & 0.022 & 0.237 & 0.788 \\
\hline
\end{tabular}




\begin{tabular}{|c|c|c|c|c|c|c|c|}
\hline & \multicolumn{5}{|c|}{ Factores } & \multirow[b]{2}{*}{ ri-t } & \multirow[b]{2}{*}{$a-i$} \\
\hline & 1 & 2 & 3 & 4 & 5 & & \\
\hline \multicolumn{8}{|l|}{ Neuroticismo $(a=0.77)$} \\
\hline BFI.14. Con frecuencia se pone tensa & 0.015 & 0.716 & 0.068 & -0.055 & -0.011 & 0.535 & 0.733 \\
\hline BFI.39. Se pone nerviosa con facilidad & -0.151 & 0.715 & -0.009 & -0.126 & 0.015 & 0.538 & 0.732 \\
\hline BFI.9. Calmada, controla bien el estrés & -0.030 & 0.673 & -0.048 & 0.147 & -0.155 & 0.528 & 0.735 \\
\hline BFI.19. Se preocupa mucho & 0.090 & 0.648 & 0.124 & -0.071 & 0.195 & 0.459 & 0.747 \\
\hline BFI.24. Es emocionalmente estable, difícil de alterar & -0.018 & 0.609 & -0.069 & 0.050 & -0.170 & 0.479 & 0.744 \\
\hline BFI.34. Mantiene la calma en situaciones tensas & -0.231 & 0.579 & -0.124 & 0.078 & -0.198 & 0.433 & 0.751 \\
\hline BFI.29. Tiene un humor cambiante & 0.084 & 0.564 & -0.075 & -0.067 & -0.155 & 0.427 & 0.755 \\
\hline BFI.4. Es depresiva. Melancólica & 0.096 & 0.485 & -0.191 & -0.186 & -0.081 & 0.377 & 0.761 \\
\hline \multicolumn{8}{|l|}{ Responsabilidad $(a=0.74)$} \\
\hline BFI.33. Hace las cosas de manera eficiente & 0.152 & -0.035 & 0.644 & 0.042 & 0.213 & 0.483 & 0.714 \\
\hline BFI.23. Tiende a ser vaga & -0.058 & -0.054 & 0.625 & 0.013 & 0.098 & 0.485 & 0.708 \\
\hline BFI.13. Es trabajadora y cumplidora. digna de confianza & 0.112 & 0.111 & 0.622 & -0.006 & 0.251 & 0.457 & 0.716 \\
\hline BFI.18. Tiende a ser desorganizada & -0.130 & -0.028 & 0.585 & -0.127 & -0.032 & 0.459 & 0.715 \\
\hline BFI.8. Es algo descuidada & -0.225 & -0.093 & 0.564 & -0.085 & -0.036 & 0.440 & 0.716 \\
\hline BFI.43. Se distrae fácilmente & -0.069 & -0.192 & 0.563 & -0.049 & -0.095 & 0.433 & 0.718 \\
\hline BFI.3. Hace un buen trabajo & 0.154 & -0.028 & 0.492 & 0.081 & 0.106 & 0.371 & 0.728 \\
\hline BFI.28. Persevera hasta terminar el trabajo & 0.144 & 0.063 & 0.470 & 0.037 & 0.210 & 0.356 & 0.730 \\
\hline BFI.38. Hace planes y los sigue cuidadosamente & 0.071 & 0.004 & 0.465 & 0.002 & -0.013 & 0.351 & 0.732 \\
\hline \multicolumn{8}{|l|}{ Extroversión $(a=0.75)$} \\
\hline BFI.21. Tiende a ser callada & -0.023 & -0.072 & -0.003 & 0.787 & 0.018 & 0.608 & 0.697 \\
\hline BFI.36. Es extrovertida. Sociable & 0.115 & -0.001 & 0.080 & 0.734 & 0.277 & 0.663 & 0.688 \\
\hline BFI.31. Es a veces tímida, inhibida & -0.028 & -0.284 & -0.002 & 0.644 & -0.113 & 0.475 & 0.723 \\
\hline BFI.6. Es reservada & -0.086 & -0.052 & -0.293 & 0.619 & 0.004 & 0.439 & 0.730 \\
\hline BFI.1. Es conversadora & 0.037 & 0.073 & 0.037 & 0.593 & 0.114 & 0.406 & 0.736 \\
\hline BFI.16. No le molesta ser el centro de atención & 0.067 & -0.009 & -0.139 & 0.496 & -0.001 & 0.400 & 0.739 \\
\hline BFI.26. No teme expresar lo que siente & 0.103 & 0.023 & 0.161 & 0.417 & 0.126 & 0.325 & 0.750 \\
\hline BFı.11. Está llena de energía & 0.212 & -0.113 & 0.230 & 0.356 & 0.292 & 0.325 & 0.750 \\
\hline \multicolumn{8}{|l|}{ Amabilidad $(a=0.68)$} \\
\hline BFI.32. Es considerada y amable con casi todo el mundo & 0.024 & -0.017 & 0.108 & 0.051 & 0.728 & 0.214 & 0.695 \\
\hline BFI.42. Le gusta cooperar con los demás & 0.111 & 0.078 & 0.113 & 0.164 & 0.654 & 0.471 & 0.635 \\
\hline BFI.22. Tiende a pensar lo mejor de la gente & 0.070 & -0.172 & -0.023 & 0.191 & 0.587 & 0.415 & 0.642 \\
\hline BFI.27. Tiende a ser cálida & 0.087 & -0.005 & 0.126 & 0.202 & 0.574 & 0.423 & 0.642 \\
\hline BFI.37. Es a veces grosera con los demás & -0.057 & -0.137 & 0.151 & -0.209 & 0.548 & 0.330 & 0.661 \\
\hline BFI.7. Ayuda desinteresadamente a los demás & 0.129 & 0.048 & 0.199 & 0.027 & 0.451 & 0.352 & 0.656 \\
\hline BFI.12. Inicia enfrentamientos o discusiones con los demás & -0.179 & -0.244 & 0.011 & -0.309 & 0.430 & 0.256 & 0.676 \\
\hline BFI.2. Tiende a encontrar fallas en los demás & -0.106 & -0.225 & -0.055 & -0.005 & 0.350 & 0.281 & 0.670 \\
\hline BFI.17. No le cuesta perdonar & 0.096 & -0.128 & -0.049 & 0.084 & 0.281 & 0.214 & 0.695 \\
\hline
\end{tabular}

Nota-. En negrita se indican los ítems agregados y en cursiva los invertidos.

Fuente: elaboración propia. 
Con respecto a la varianza total explicada, la misma fue de $39.52 \%$, desglosándose en $8.93 \%$ para Apertura, $8.06 \%$ para Responsabilidad, $7.88 \%$ para Neuroticismo, 7.53 \% para Extroversión y 7.11 \% para Amabilidad.

Luego de analizadas la validez y la consistencia interna de las dimensiones que conforman al $\mathrm{BFl}$, se procedió a evaluar las diferencias según el sexo en los cinco factores y las relaciones entre estos y la edad de los participantes. Con respecto al sexo, se observaron diferencias estadísticamente significativas en los factores Amabilidad $\left(t_{(535)}=\right.$ $\left.-3.725 ; p<0.001 ; M_{\text {Hombres }}=3.57 ; M_{\text {Mujeres }}=3.77\right)$ y Neuroticismo $\left(t_{(534)}=-4.993 ; p<0.001 ; M_{\text {Hombres }}\right.$ $=2.89 ; M_{\text {Mujeres }}=3.24$ ), siendo las mujeres las que obtienen mayores puntajes que los hombres en ambos factores. En cuanto a la edad, se observaron relaciones estadísticamente significativas solo con los factores Neuroticismo $(r=-0.11 ; p<0.01)$ y Responsabilidad $(r=0.18 ; p<0.001)$.
Por último, se analizaron las relaciones entre los cinco factores de personalidad y la autoestima, siendo significativas con Amabilidad $(r=0.28 ; p<0.01)$, Extraversión $(r=0.23 ; p<0.01)$, Responsabilidad $(r=0.34 ; p<0.01)$, Neuroticismo $(r=-0.36 ; p<0.01)$ y Apertura a la experiencia $(r=0.15 ; p<0.01)$.

\section{Factores de orden superior de la personalidad}

Para evaluar lo planteado inicialmente por Digman (1997) y retomado posteriormente por De Young et al. (2002), se procedió a realizar un Análisis Factorial Confirmatorio con los cinco factores de personalidad transformados e indagar si el modelo de dos factores de orden superior, estabilidad y plasticidad, es el que mejor se ajusta a los datos (Figura 1). Además, se analizaron las relaciones entre la Estabilidad $(r=0.46 ; p<0.01)$ y Plasticidad $(r=0.25 ; p<0.01)$ con respecto a la autoestima, siendo ambas relaciones estadísticamente significativas y positivas.

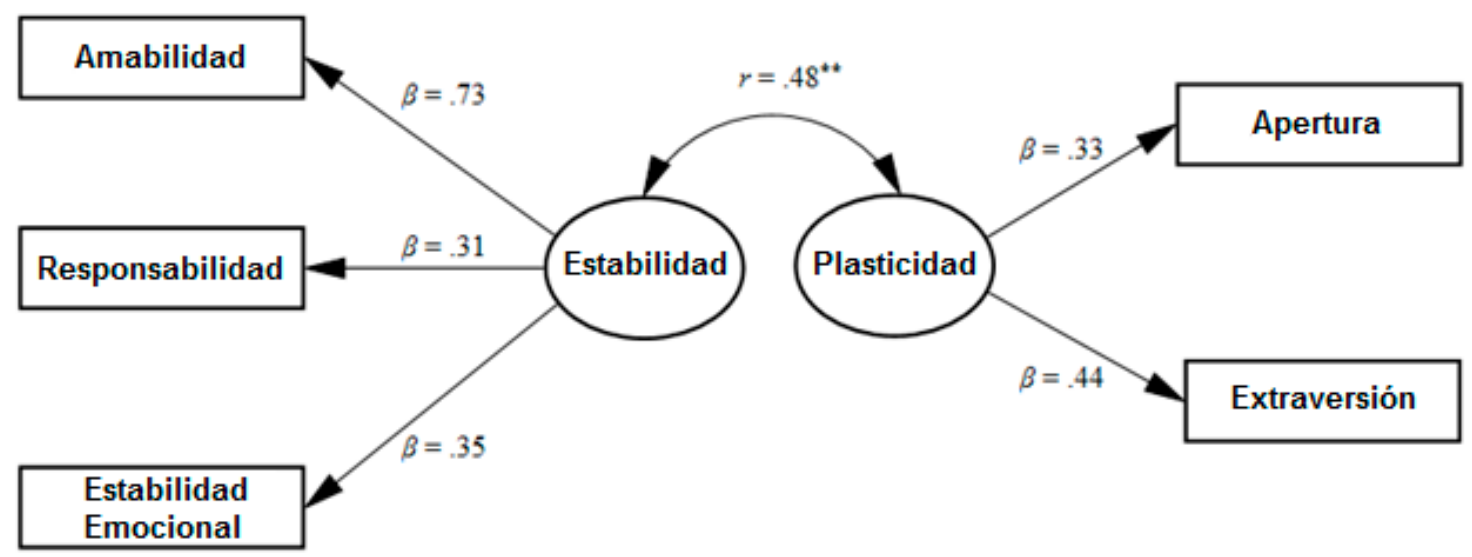

Figura 1. Análisis factorial confirmatorio de Estabilidad y Plasticidad

${ }^{* *} p<0.001$.

Nota. $-\mathrm{X}_{(4)}=4.698 ; p=0.320 ; \mathrm{X}^{2} / d f=1.17 ; \Delta \mathrm{GFI}=0.98 ; \Delta=0.99 ;$ CFI $=0.99 ;$ RMSEA $=0.017$.

Fuente: elaboración propia. 


\section{Discusión}

En primer lugar, los resultados obtenidos indicaron adecuadas propiedades psicométricas para la adaptación y validación de la versión original del Inventario de los Cinco Grandes Factores de Personalidad (BFl; John et al., 1991). Tras el reemplazo de cuatro ítems y consistentemente con los resultados obtenidos en la versión original del inventario, se pudo replicar la estructura de 44 ítems agrupados en cinco factores que explicaron el $39.5 \%$ de la varianza total. La misma solución factorial fue hallada en diferentes estudios llevados a cabo en múltiples contextos (Benet-Martínez y John, 1998; John et al., 2008). En cuanto a la consistencia interna de las dimensiones del instrumento, si bien los indicadores para cada una de los factores fueron inferiores a los obtenidos por John et al. (1991), son similares o mayores a los hallados en distintas investigaciones que utilizaron la técnica en el contexto argentino (Castro Solano, 2005; Castro Solano y Casullo, 2001).

Tal y como fue sugerido por diferentes autores (Robins et al., 2001; Shackelford y Michalski, 2011), en el presente estudio se observaron asociaciones entre todos los factores del BFI y la autoestima. No obstante, al igual que lo sugiere la literatura previa sobre el tema, las asociaciones más fuertes fueron las observadas con los factores de Neuroticismo (Costa et al., 1992; Judge et al., 2002) y Responsabilidad (Costa et al., 1992; Digman, 1997). En el primer caso, dado que como fue mencionado el Neuroticismo implica una elevada predisposición a experimentar emociones negativas (Lingjaerde et al., 2001), esta tendencia también predispondría a los sujetos a percibirse negativamente a sí mismos resultando en una baja autoestima (Judge et al., 2002). Por el contrario, en la medida en que la autoestima refleja la evaluación global de una persona de sus cualidades, se deduce que los individuos con alta responsabilidad también se atribuirían características deseables a ellos mismos, ya sea porque efectivamente se ven como personas capaces de controlar sus impulsos, actuar con metas claras, planificar, organizar y llevar adelante proyectos e ideas (Lingjaerde et al., 2001), o bien por su tendencia a presentarse de un modo socialmente aceptable (Digman, 1997). No obstante, el papel de la deseabilidad social en la relación entre los rasgos de personalidad y autoestima no ha sido suficientemente explorado en la literatura sobre la temática (Paulhus y Reid, 1991).

Además, diferentes autores sugieren la existencia de diferencias sistemáticas según la edad en los rasgos de personalidad, tanto en la adolescencia como en la adultez (e.g., Lucas y Donnellan, 2009; Srivastava et al., 2003). Al igual que en el estudio desarrollado por Soto et al. (2008), en el presente estudio se halló una tendencia positiva en las relaciones entre la edad, la Amabilidad y la Responsabilidad. En cuanto al sexo, se observaron diferencias estadísticamente significativas en los factores Amabilidad y Neuroticismo, tal como sugiere la literatura específica (Chapman et al., 2007). Las relaciones obtenidas con la autoestima y la edad, como las diferencias por sexo, proporcionan validez de criterio para el $\mathrm{BF}$ al replicarse lo que sistemáticamente se observa en la literatura sobre el tema.

Por otra parte, para indagar si lo sugerido inicialmente por Digman (1997) y posteriormente por De Young et al. (2002) acerca de la presencia de dos factores de orden superior de la personalidad se realizó en el presente estudio por medio de un AFC. Los resultados indicaron que tal como se planteó previamente (Digman, 1997; De Young et al., 2002), la solución de dos factores de orden superior resultó sumamente robusta. Además, retomando los planteamientos de De Young et al. (2002), se pudo observar que en el modelo obtenido en el presente estudio, ambos factores son complementarios ya que presentaron fuertes asociaciones entre sí. Esto indicaría que efectivamente se puede pensar en una retroalimentación de estos factores, independientemente del contexto en el que se evalúe. En otras palabras, las personas requieren plasticidad para alcanzar estabilidad ante situaciones nuevas y esta última refuerza a la primera cuando se trata de enfrentar nuevos desafíos.

De modo similar a lo que se observó en estudios previos (Erdle et al., 2009; Erdle, 2013; Simsek, 2012), la estabilidad y la plasticidad se asociaron 
positivamente a la autoestima. Esto es consistente con lo observado por Erdle et al. (2009) y Simsek (2012), reforzando la idea de que individuos con mayor estabilidad y plasticidad no solo se sienten más conformes con su respuesta a diferentes situaciones, sino que también reciben refuerzos positivos por parte del entorno y esto incrementa sus niveles de autoestima.

El presente estudio realiza diferentes aportes para el análisis de la personalidad en el contexto argentino. En primer lugar, proporciona una técnica de evaluación válida y confiable con indicadores psicométricos mejor adaptados al contexto que las anteriores evaluaciones desarrolladas (Castro Solano, 2005; Castro Solano y Casullo, 2001). En segundo lugar, reafirma el adecuado funcionamiento del instrumento tras dar cuenta de su validez de criterio a partir de las relaciones con variables que la literatura sugiere como asociadas. En tercer lugar, atendiendo a lo sugerido por diferentes autores (Digman, 1997; De Young et al., 2002) y teniendo en cuenta que no había sido evaluado en el contexto argentino, se encuentra evidencia a favor de una estructura factorial compuesta por dos factores de orden superior, que además se vuelve más robusta a partir de sus relaciones con la variable autoestima.

No obstante, pese a estos aportes, cabe destacar que es necesario continuar trabajando en desarrollos teóricos y empíricos sobre la personalidad en el contexto argentino, que permitan comprender de un modo más inclusivo un fenómeno complejo. En esta línea, una de las principales limitaciones del presente estudio radica en haber trabajado solo con población de estudiantes universitarios, por lo que se recomienda que futuras investigaciones analicen dicho fenómeno y sus relaciones con las variables mencionadas, en muestras tanto de adultos como de adolescentes o niños. Además, resulta necesario continuar evaluando, por ejemplo, el efecto mediador de variables como el sexo, la etnia, el nivel socioeconómico y la nacionalidad en la relación con los rasgos de personalidad y la autoestima. Por último, se sugiere indagar acerca de los efectos de la deseabilidad social como mediadora de alguna de las relaciones entre la personalidad y el resto de las variables propuestas.

\section{Referencias}

Allport, G. W. y Odbert, H. S. (1936). Traitnames: A psycho-lexical study. Psychological Monographs, 47(1), 171 . https://doi. org/10.1037/h0093360

Ashton, M. C., Lee, K., Goldberg, L. R. y de Vries, R. E. (2009). Higher order factors of personality: do they exist? Personality and Social Psychology Review, 13(2), 79-91. https://doi. org/10.1177/1088868309338467

Bäckström, M., Björklund, F. y Larsson, M. R. (2009). Five-factor inventories have a major general factor related to social desirability which can be reduced by framing items neutrally. Journal of Research in Personality, 43(3), 335-344. https://doi.org/10.1016/j.jrp.2008.12.013

Benet-Martinez, V. y John, O. P. (1998). Los Cinco Grandes across cultures and ethnic groups: Multitrait multimethod analyses of the Big Five in Spanish and English. Journal of Personality and Social Psychology, 75, 729-750.

Blackburn, R., Renwick, S. J., Donnelly, J. P. y Logan, C. (2004). Big five or big two? Superordinate factors in the NEO five factor inventory and the antisocial personality questionnaire. Personality and Individual Differences, 37(5), 957-970. https://doi.org/10.1016/j.paid.2003.10.017

Buss, D. (1995). Psychological sex differences: Origins through sexual selection. American Psychologist, 50(3), 164-168. https://doi. org/10.1037/0003-066X.50.3.164

Caprara, G. V., Schwartz, S., Capanna, C., Vecchione, M. y Barbaranelli, C. (2006). Personality and politics: Values, traits, and political choice. Political Psychology, 27(1), 1-28. https://doi. org/10.1111/j.1467-9221.2006.00447.x

Carver, C. S. y Scheier, M. F. (2004). Perspectives on personality (5th ed.). Boston: Allyn \& Bacon.

Caspi, A. y Roberts, B. (2001). Personality Development Across the Life Course: The Argument for Change and Continuity. Journal 
of Psychological Inquiry, 12(2), 49-66. https:// doi.org/10.1207/S15327965PLI1202_01

Castro Solano, A. (2005). Técnicas de evaluación psicológica en los ámbitos militares: Motivación, valores y liderazgo. Buenos Aires: Paidos.

Castro Solano, A. y Casullo, M. M. (2001). Rasgos de personalidad, rendimiento académico y bienestar autopercibido en adolescentes argentinos. Interdisciplinaria, 18, 65-85.

Cattell, R. B. (1943). The description of personality: Basic traits resolved into clusters. The Journal of Abnormal and Social Psychology, 38(4), 476506. https://doi.org/10.1037/h0054116

Chan, W., Mccrae, R. R., De Fruyt, F., Jussim, L., Löckenhoff, C. E., . . . Terracciano, A. (2012). Stereotypes of age differences in personality traits: Universal and accurate? Journal of Personality and Social Psychology, 103(6), 1050-1066. https://doi.org/10.1037/a0029712

Chapman, B., Lyness, J. M., Duberstein, P. R. y Sörensen, S (2007). Gender difference in five factor model personality traits in an elderly cohort. Personality and Individual Differences, 43(6), 1594-1603. https://doi.org/10.1016/j. paid.2007.04.028

Costa, P. T. y McCrae, R. R. (1980). Still stable after all these years: personality as a key to some issues in adulthood and old age. En P.B. Baltes y O.G. Brim, (Eds.). Life span development and behaviour (3rd. ed.) (pp. 65-102). New York: Academic Press.

Costa, P. T. y McCrae, R. R. (1992). Revised NEO Personality Inventory (NEO PI-R) and NEO Five Factor Inventory (NEO-FFI) professional manual. Odessa: Psychological Assessment Resources.

Cupani, M. (2009). El cuestionario de Personalidad IPIP-FFM: Resultados preliminares de una adaptación en una muestra de preadolescentes argentinos. Perspectivas en Psicología, 6, 51-58.

Cupani, M., Pilatti, A., Urrizaga, A., Chincolla, A. y Richaud de Minzi, M. (2014). Inventario de personalidad IPIP-NEO: estudios preliminares de adaptación al español en estudiantes argentinos. Revista Mexicana de Investigación en Psicología 6(1), 55-73.

DeYoung, C. G. (2006). Higher-order factors of the Big Five in a multi-informant sample. Journal of Personality and Social Psychology, 91(6), 1138-1151. https://doi. org/10.1037/0022-3514.91.6.1138

DeYoung, C. G., Peterson, J. B. y Higgins, D. M. (2002). Higher-order factors of the Big Five predict conformity: Are there neuroses of health? Personality and Individual Differences, 33(4), 533-552. https://doi.org/10.1016/ S0191-8869(01)00171-4

Digman, J. M. (1997). Higher-order factors of the Big Five. Journal of Personality and Social Psychology, 73(6), 1246-1256.

Eagly, A. H. (1987). Sex differences in social behavior: A social-role interpretation. Hillsdale, NJ: Erlbaum.

Ekehammar, B., Akrami, N., Gylje, M. y Zakrisson, I. (2004). What matters most to prejudice: Big Five personality, Social Dominance Orientation, or Right-Wing Authoritarianism? European Journal of Personality, 18(6), 463-482. https:// doi.org/10.1002/per.526

Erdle, S. (2013). Self-esteem and higher-order factors of personality: A reply to Simsek. Personality and Individual Differences, 54(4), 545-546. https://doi.org/10.1016/j.paid.2012.10.030

Erdle, S., Gosling, S. D. y Potter, J. (2009). Does self-esteem account for the higher-order factors of the Big Five?. Journal of Research in Personality, 43(5), 921-922. https://doi. org/10.1016/j.jrp.2009.04.012

Feingold, A. (1994). Gender differences in personality: A meta-analysis. Psychological Bulletin, 116(3), 429-456. https://doi. org/10.1037/0033-2909.116.3.429

Genise, G. (2015). Relación entre el estilo personal del terapeuta, estilo de apego y factores 
de personalidad del terapeuta. Psicodebate. Psicología, Cultura y Sociedad, 15(1), 9-22. http://dx.doi.org/10.18682/pd.v15i1.481

Goldberg, L. R. (1999). A broad-bandwidth, publicdomain, personality inventory measuring the lower-level facets of several Five-Factor models en Mervielde y Col. Personality Psychology in Europe, 7, 7-28.

Goldberg, L. R. y Kilkowski, J. M. (1985). The prediction of semantic consistency in self-descriptions: Characteristics of persons and of terms that affect the consistency of responses to synonym and antonym pairs. Journal of Personality and Social Psychology, 48(1), 8298. https://doi.org/10.1037/0022-3514.48.1.82

Goldberg, L. R. y Rosolack, T. K. (1994). The Big Five factor structure as an integrative framework: An empirical comparison with Eysenck's P-E-N model. En C. F. Halverson, Jr., G. A. Kohnstamm y R. P. Martin (Eds.), The developing structure of temperament and personality from infancy to adulthood (p. 7-35). Lawrence Erlbaum Associates, Inc.

Góngora, V. y Casullo, M. (2009). Factores protectores de la salud mental: un estudio comparativo sobre valores, autoestima e inteligencia emocional en población clínica y población general. Interdisciplinaria, 26(2), 183-205.

Hirsh, J. B. (2010). Personality and environmental concern. Journal of Environmental Psychology, 30(2), 245-248. https://doi.org/10.1016/j. jenvp.2010.01.004

Hull, D. M. y Beaujean, A. A. (2011). Higher order factors of personality in Jamaican young adults. Personality and Individual Differences, 50(6), 878-882. https://doi.org/10.1016/j. paid.2011.01.013

John, O. P. (1990). The "Big Five" factor taxonomy: Dimensions of personality in the natural language and questionnaires. En L. A. Pervin (Ed.) Handbook of personality: Theory and research (pp. 66-100). New York: Guilford Press.
John, O. P., Donahue, E. M. y Kentle, R. L. (1991). The Big Five Inventory-Versions $4 a$ and 54. Berkeley, CA: University of California, Berkeley, Institute of Personality and Social Research.

John, O. P., Naumann, L. P. y Soto, C. J. (2008). Paradigm Shift to the Integrative Big-Five Trait Taxonomy: History, Measurement, and Conceptual Issues. In O. P. John, R. W. Robins y L. A. Pervin (Eds.), Handbook of personality: Theory and research (pp. 114-158). New York, NY: Guilford Press.

John, O. P., Robins, R. W. y Pervin, L. A. (Eds.). (2008). Handbook of personality: Theory and research ( $3 a$ ed.). New York, Ny: The Guilford Press.

John, O. P. y Srivastava, S. (1999). The Big Five trait taxonomy: History, measurement, and theoretical perspectives. En L. A. Pervin y O. P. John (Eds.), Handbook of personality: Theory and research (2nd ed., pp. 102-138). New York, NY: The Guilford Press.

Judge, T. A., Erez, A., Bono, J. E. y Thoresen, C. J. (2002). Are measures of self- esteem, neuroticism, locus of control, and generalized self-efficacy indicators of a common core construct? Journal of Personality and Social Psychology, 83(3), 693-710. https://doi. org/10.1037/0022-3514.83.3.693

Lee, A. y Hankin, B. L. (2009). Insecure attachment, dysfunctional attitudes, and low selfesteem predicting prospective symptoms of depression and anxiety during adolescence. Journal of Clinical Child and Adolescent Psychology, 38(2), 219-231. https://doi. org/10.1080/15374410802698396

Lingjaerde, O., Regine Foreland, A. y Engvik, H. (2001). Personality structure in patients with winter depression, assessed in a depression free state according to the five - factor model of personality. Journal of Affective Disorders, 62(3), 165 - 174. https://doi.org/10.1016/ S0165-0327(99)00183-4 
Lucas, R. E. y Donnellan, M. B. (2009). Age differences in personality: Evidence from a nationally representative Australian sample. Developmental Psychology, 45(5), 1353- 1363. https://doi.org/10.1037/a0013914

Macmillan, M. (2000). An Odd Kind of Fame: Stories of Phineas Gage. Cambridge, MA: мIт Press.

McCrae, R. R. y Costa Jr., P.T. (2012). Personality in adulthood: A five-factor theory perspective. Guilford Press.

Mischel, W. (1979). Introduction to Personality (6th ed). Harcourt Brace: Fort Wort.

Muñiz, J. y Hambleton, R. K. (2000). Adaptación de los tests de unas culturas a otras. Metodología de las Ciencias del Comportamiento, 2(2), 129-149.

Musek, J. (2007). A general factor of personality: Evidence for the Big One in the five-factor model. Journal of Research in Personality, 41(6), 12131233. https://doi.org/10.1016/j.jrp.2007.02.003

Paulhus, D. L. y Reid, D. B. (1991). Enhancement and denial in socially desirable responding. Journal of Personality and Social Psychology, 60(2), 307317. https://doi.org/10.1037/0022-3514.60.2.307

Penke, L., Denissen, J. J. A. y Miller, G. F. (2007). The evolutionary genetics of personality. European Journal of Personality, 21(5), 549587. https://doi.org/10.1002/per.629

Phelps, B. J. (2000). Dissociative identity disorder: The relevance of behavior analysis. The Psychological Record, 50(2), 235-249.

Rentfrow, P. J., Gosling, S. D. y Potter, J. (2008). A Theory of the Emergence, Persistence, and Expression of Geographic Variation in Psychological Characteristics. Perspectives on Psychological Science, 3(5), 339-369. https:// doi.org/10.1111/j.1745-6924.2008.00084.x

Robins, R. W., Hendin, H. M. y Trzesniewski, K. (2001). Measuring global self- esteem: Construct validation of a single-item measure and the
Rosenberg Self- Esteem Scale. Personality and Social Psychology Bulletin, 27, 151-161. https:// doi.org/10.1177/0146167201272002

Robson, P. (1989). Development of a new self-report questionnaire to measure self esteem. Psychological Medicine, 19(2), 513-518. https:// doi.org/10.1017/S003329170001254X

Rosenberg, M. (1965). Society and the adolescent self-image. Princeton, NJ: Princeton University Press.

Rushton, J. P. e Irwing, P. (2008). A general factor of personality from two meta-analyses of the Big Five: Digman (1997) and Mount, Barrick, Scullen, and Rounds (2005). Personality and Individual Differences, 45(7), 679-683. https:// doi.org/10.1016/j.paid.2008.07.015

Rushton, J. P. e Irwing, P. (2009). A general factor of personality in 16 sets of the Big Five, the Guilford-Zimmerman Temperament Survey, California Psychological Inventory, and Temperament and Character Inventory. Personality and Individual Differences, 47(6), 558-564. https://doi.org/10.1016/j. paid.2009.05.009

Rushton, J. P., Bons, T. A. y Hur, Y.-M. (2009). The genetics and evolution of a general factor of personality. Journal of Research in Personality, 43(3), 1173-1185. https://doi.org/10.1016/j. jrp.2009.01.005

Santrock, J.W. (2008). Adolescence: Twelfth edition. McGraw-Hill Higher Education.

Saroglou, V. y Muñoz García, A. (2008). Individual differences in religion and spirituality: An issue of personality traits and/ or values. Journal for the Scientific Study of Religion, 47(1), 83-101. https://doi. org/10.1111/j.1468-5906.2008.00393.x

Schmitt, D. P., Allik, J., McCrae, R. R. y BenetMartínez, V. (2007). The geographic distribution of Big Five personality traits patterns and profiles of human self-description across 56 nations. Journal of Cross-Cultural 
Psychology, 38(2), 173-212. https://doi. org/10.1177/0022022106297299

Schmitt, D., Realo, A., Voracek, M. y Allik, J. (2008). Why Can't a Man Be More Like a Woman? Sex Differences in Big Five Personality Traits Across 55 Cultures. Journal of Personality and Social Psychology, 94(1), 168-182. https://doi. org/10.1037/0022-3514.94.1.168

Shackelford, T. K. y Michalski, R. L. (2011). Personality and self-esteem in newlyweds. Personality and Individual Differences, 51(7), 870-872. https:// doi.org/10.1016/j.paid.2011.07.018

Şimşek, Ö. F. y Koydemir, S. (2012). Linking Metatraits of the Big Five to Well-Being and Ill-Being: Do Basic Psychological Needs Matter? Social Indicators Research, 112(1) 1-18. https:// doi.org/10.1007/s11205-012-0049-1

Simsek, D. (2012). Identity formation of Cypriot Turkish, Kurdish and Turkish young people in London in a transnational context. (Tesis Doctoral no publicada / Unpublished Doctoral thesis, City University London).

Snygg, D. (1949). Predicting the behavior of individuals. Canadian Journal of Psychology/Revue canadienne de psychologie, 3(1), 19-29. https:// doi.org/10.1037/h0084078

Soto, C. J., John, O. P., Gosling, S. D. y Potter, J. (2008). The developmental psychometrics of Big-Five self-reports: Acquiescence, factor structure, coherence, and differentiation from ages 10 to 20. Journal of Personality and Social Psychology, 94(4), 718-737. https://doi. org/10.1037/0022-3514.94.4.718

Srivastava, S., John, O. P., Gosling, S. D. y Potter, J. (2003). Development of personality in early and middle adulthood: Set like plaster or persistent change? Journal of Personality and Social Psychology, 84(5), 1041-1053. https:// doi.org/10.1037/0022-3514.84.5.1041

Terracciano, A., Costa Jr, P. T. y McCrae, R. R. (2006). Personality plasticity after age 30 . Personality and Social Psychology Bulletin, 32(8), 999-1009. https://doi.org/10.1177/0146167206288599

Van Aken M. A., Denissen, J. J., Branje, S. J., Dubas, J. S. y Goossens, L. (2006). Midlife concerns and short-term personality change in middle adulthood. European Journal of Personality, 20(6), 497-513. https://doi.org/10.1002/per.603

Vecchione, M., Alessandri, G., Barbaranelli, C. y Caprara, G. (2011). Higher-order factors of the big five and basic values: Empirical and theoretical relations. British Journal of Psychology, 102(3), 478-498. https://doi. org/10.1111/j.2044-8295.2010.02006.x

Woods, S. A. y Hardy, C. (2011). The higher-order factor structures of five personality inventories. Personality and Individual Differences, 52(4), 552-558. https://doi.org/10.1016/j. paid.2011.11.001 